\title{
Delivery of siRNA into the blood-brain barrier: recent advances and future perspective
} "Practically speaking, it may be easier to establish techniques to deliver siRNA into the
blood-brain barrier rather than across it..."

Keywords: blood-brain barrier $\approx$ brain capillary endothelial cell $\approx$ neurological disease $\approx$ siRNA delivery

The development of gene-silencing therapy for neurological diseases has placed great importance on the delivery of siRNA from the blood into the brain across the blood-brain barrier (BBB). Increasingly, gene-silencing therapy in the $\mathrm{BBB}$ itself is being considered because the $\mathrm{BBB}$ is associated with the pathophysiologies of many major diseases such as brain ischemia, multiple sclerosis and neurodegenerative disorders. Practically speaking, it may be easier to establish techniques to deliver siRNA into the $\mathrm{BBB}$ rather than across it, because there has been an accumulation of knowledge concerning the dynamic interactions between the blood circulation and brain capillary endothelial cells (BCECs), the chief components of the BBB. The purpose of this Editorial is to explain the recent advances in siRNA delivery into the BBB and to express some opinions on future research in this field.

\section{The blood-brain barrier}

The BBB is composed mainly of BCECs and has pericytes, astrocyte foot processes and nerve endings terminating at the capillary surfaces [1]. The BBB is a unique structure in the CNS that is both a physical barrier, resulting from the presence of endothelial tight junctions, and a transport barrier, resulting from selective membrane transporters and vesicular trafficking via BCECs [2]. It is generally considered that for compounds to cross the BBB they should have a molecular weight of less than $400 \mathrm{Da}$ and be lipophilic [1].

The establishment of a technique to deliver siRNA across the BBB is expected to result in the development of gene-silencing therapies for a variety of neurological diseases; however, delivering siRNA across the $\mathrm{BBB}$ remains a challenge since siRNA molecules weigh more than
10,000 Da and are not lipophilic. So far, many technical developments have been reported such as the chemical modification of liposomal complexes with siRNA, the direct conjugation of siRNA to ligands for the BBB and the use of cellpenetrating peptides that can be noncovalently complexed or covalently linked to siRNA [3]. The precise mechanisms of these delivery strategies remain to be elucidated; therefore, most preclinical and clinical studies on the delivery of siRNA into the central nervous system have used the invasive techniques of intracerebral, intracerebroventricular or intrathecal injection into or near the targeted tissues [3]. To our knowledge, no trials have used intravascular injections to deliver siRNA across the BBB.

\section{Pathophysiologies at the BBB}

It is noteworthy that some major neurological diseases have pathophysiologies at the BBB itself, especially at BCECs, apart from those at neurons or glia $[4,5]$. BCECs express a range of molecules that can be targeted for silencing.

Inflammatory cell-adhesion molecules such as ICAM-1, VCAM-1, and the selectins are responsible for secondary neuronal injury after reperfusion in brain ischemia [6]. The matrixdegrading metalloproteinases (MMPs), particularly MMP-9, are involved in the process of neuroinflammation associated with the ischemic condition [7]. In an in vitro BBB model, silencing of MMP-9 by siRNA was reported to enhance $\mathrm{BBB}$ integrity [8].

Inflammatory cell-adhesion molecules are also related to the pathophysiologies of multiple sclerosis [9]. They help activated leukocytes cross the $\mathrm{BBB}$ and enter the brain, resulting in the initiation of immune-mediated demyelination [9]. The therapeutic concept of inhibiting

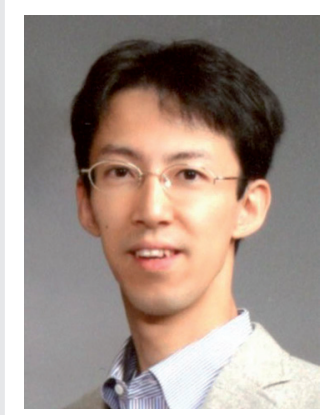

Hiroya Kuwahara

Department of Neurology \&

Neurological Science, Graduate

School, Tokyo Medical \& Dental University

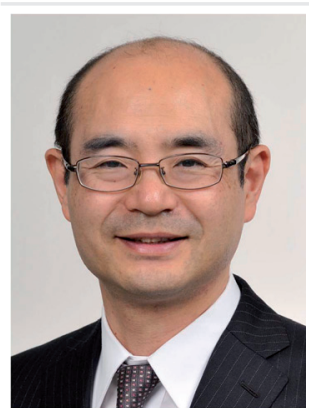

Takanori Yokota

Author of correspondence:

Department of Neurology \&

Neurological Science, Graduate

School, Tokyo Medical \& Dental

University, I-5-45, Yushima,

Bunkyo-ku, Tokyo II3-85I9, Japan

Tel.: +8I 358035234

Fax: +81 358030169

E-mail: tak-yokota.nuro@tmd.ac.jp

Author affiliations continue

overleaf... 


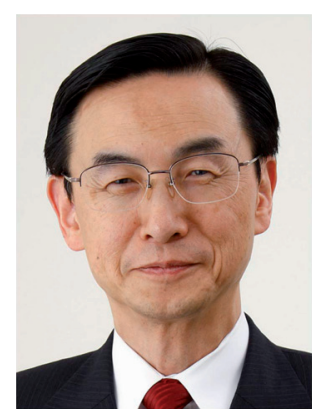

Hidehiro Mizusawa

Department of Neurology \& Neurological Science, Graduate School, Tokyo Medical \& Dental University the adhesion of leukocytes to BCECs in multiple sclerosis is currently being employed in the clinical setting; natalizumab, a monoclonal antibody against very late antigen- 4 in leukocytes, inhibits leukocytes from binding to VCAM-1 (the receptor of very late antigen-4) in BCECs [10].

In Alzheimer's disease, the receptor for advanced glycation endproducts (RAGE) in BCECs mediates the transport of the neurotoxic amyloid- $\beta$ peptide from the blood into the brain, leading to oxidative stress and neuroinflammation [11]. Expression of RAGE in BCECs is approximately 2.5-times higher in Alzheimer's disease patients than in age-matched controls, and inhibition of RAGE in BCECs may alleviate the disease pathology [12].

The diseases mentioned above have a high prevalence in most developed countries, and effective therapies remain to be established. As clinicians in neurology, we wish to emphasize that the BBB itself possesses great potential as an important target for gene-silencing therapy for these diseases.

\section{Delivery of siRNA into the BBB}

Few attempts of using the concept of gene-silencing at the BBB itself have been reported. We first reported the delivery of siRNA into BCECs via a hydrodynamic injection technique [13], and the same strategy was adopted in subsequent studies $[14,15]$. One possible delivery mechanism of this technique is that rapid loading of an extremely large volume of solution results in a considerable increase in hydrostatic pressure in the brain capillaries. Another possible explanation is that rapid injection of a large volume of solution prevents the solution from mixing with the serum containing RNase, thereby keeping the concentration of siRNA high in the brain capillaries. However, hydrodynamic injection cannot be used clinically because it can result in injury to various organs and tissues. There is, therefore, a need to develop an alternative strategy that is clinically feasible.

We recently reported the efficient delivery of siRNA into BCECs along with endogenous lipoprotein [16]. A cholesterol-conjugated siRNA was incorporated into extracted endogenous highdensity lipoproteins and then intravenously injected into mice. Cholesterol-conjugated siRNA was not delivered into neurons or glia, but was successfully delivered into BCECs by receptor-mediated uptake. Because cholesterol is taken up into BCECs along with lipoproteins but generally does not pass through the BBB, cholesterol conjugation might be an effective strategy to deliver siRNA into BCECs. To date, our report is the only one to have demonstrated a technique that enables siRNA to be delivered into BCECs and be used in a clinical setting, although improvements would need to be made before this could be done [16].

\section{Future perspective}

The biggest challenge for the efficient delivery of siRNA into BCECs is the enhancement of delivery through the reduction of tropism to the liver, secretion from the kidney and uptake into the reticuloendothelial system, such as the spleen, lymph node and bone marrow. In addition to optimizing the size, charge and lipophilicity of siRNA vectors, other possible improvements include modification of their surfaces, for example, by binding them to ligands that interact with the BCEC membrane proteins, or by coating them with polyethylene glycol to increase retention in the blood. Recently, Chen et al. succeeded in delivering an increased amount of a gene to BCECs by inserting polypeptides, selected from a phage library by in vivo panning, into the binding site of an adeno-associated virus vector to its receptor [17]. Increase in affinity to BCECs and decrease in distribution to other organs/tissues will be helpful in reducing the dosage and side effects of siRNA.

It is also important to investigate the therapeutic utility by using model animals for the neurological diseases mentioned above. Our previous reports regarding a hydrodynamic injection technique [13] and a lipoprotein vector [16] demonstrate only the proof-of-principle of siRNA delivery into BCECs by targeting organic anion transporter 3, which is exclusively expressed in BCECs in the brain and is not recognized as a pathological molecule in any neurological disease. If gene-silencing therapy in BCECs is actually demonstrated to be effective by in vivo delivery of siRNA in some disease models, research in this field is expected to be remarkably facilitated.

We believe that techniques to deliver siRNA into BCECs could be further developed into techniques to deliver siRNA into the brain across the BBB. Theoretically, delivery across the BBB can be achieved by using either the intercellular or intracellular route. At the BBB, there is a unique intercellular junctional complex without fenestrations that is formed from tight junctions, adherence junctions and basement membranes. Intercellular delivery across the BBB would 
probably be accompanied by disruption of this junctional complex, leading to nonspecific entry of serum proteins and agents into the brain and subsequent toxicity for neurons and glia; this toxicity should be avoided.

Intracellular delivery across the BBB could result from the establishment of a method to deliver molecules into BCECs. Knowledge of the physiological transport mechanisms present at the BBB has increased remarkably [2]. A wide variety of transporters contribute to the selective influx into the brain of energy resources, amino acids, hormones and organic ions [2]. For example, glucose transporter-1, which is abundantly expressed in BCECs [18], contributes to the transport of $\mathrm{D}$-glucose and vitamin $\mathrm{C}$, and may work as a carrier of siRNA. Similarly, it might be possible to use amino acid transporters, monocarboxylic acid transporters or thyroid hormone transporters. However, there may be strict limitations on the structure of substrates, and it could therefore be difficult for siRNA to enter the brain via these transporters.

The unique endocytosis/transcytosis at the BBB involves a receptor-mediated mechanism (cargo such as transferrin, insulin and lipoprotein) and an adsorptive-mediated mechanism (cargo such as cationic proteins and cell-penetrating peptides) [2]. Utilization of these endocytosis/transcytosis systems may be an alternative strategy for the effective and safe delivery of macromolecules such as siRNA [19]. Short peptides derived from the rabies virus glycoprotein, which bind to the acetylcholine receptor, have been used to deliver siRNA across the BBB [20]. Other candidate ligands that can be used include the low-density lipoprotein receptor (LDLR)binding domain of apolipoprotein $\mathrm{B}$ (the ligand for LDLR) [21], Angiopep-2 (the ligand for LDLR-related protein-1) [22] or the nontoxic form of the diphtheria toxin receptor, CRM197 (the ligand for diphtheria toxin receptor) [23]. To achieve delivery across the BBB, it will be necessary for the cargo to stay in the endosome during intracellular trafficking. Even if the cargo can reach the abluminal membrane of BCECs, there remain at least two hurdles to overcome: the mechanism of detaching the cargo from the BCECs and the passage through the basement membrane. Accumulating knowledge of the biology of the BBB is expected to be helpful in the establishment of a method of delivering siRNA across the BBB.

In conclusion, the pursuit of delivering siRNA into the BBB to develop gene-silencing therapy for diseases involving BCECs is noteworthy. Research from this perspective might result in important knowledge for the development of techniques to deliver siRNA from the blood into the brain across the BBB.

\section{Financial \& competing interests disclosure}

This work was supported by grants from the Ministry of Health, Labour and Welfare of Japan (\#2212070 and \#2212148) and a grant from the Ministry of Education, Science and Culture of Japan (\#20659138). The authors have no other relevant affiliations or financial involvement with any organization or entity with a financial interest in or financial conflict with the subject matter or materials discussed in the manuscript apart from those disclosed.

No writing assistance was utilized in the production of this manuscript.

\section{References}

1 Pardridge WM. Blood-brain barrier genomics. Stroke 38(2), 686-690 (2007).

2 Abbott NJ, Patabendige AA, Dolman DE, Yusof SR, Begley DJ. Structure and function of the blood-brain barrier. Neurobiol. Dis. 37(1), 13-25 (2010).

3 Mathupala SP. Delivery of small-interfering RNA (siRNA) to the brain. Expert Opin. Ther. Pat. 19(2), 137-140 (2009).

4 Zlokovic BV. The blood-brain barrier in health and chronic neurodegenerative disorders. Neuron 57(2), 178-201 (2008).

5 Neuwelt EA, Bauer B, Fahlke C et al. Engaging neuroscience to advance translational research in brain barrier biology. Nat. Rev. Neurosci. 12(3), 169-182 (2011).
6 Frijns CJ, Kappelle LJ. Inflammatory cell adhesion molecules in ischemic cerebrovascular disease. Stroke 33(8), 2115-2122 (2002).

7 Jin R, Yang G, Li G. Molecular insights and therapeutic targets for blood-brain barrier disruption in ischemic stroke: critical role of matrix metalloproteinases and tissue-type plasminogen activator. Neurobiol. Dis. 38(3), 376-385 (2010).

8 Bonoiu A, Mahajan SD, Ye L et al. MMP-9 gene silencing by a quantum dot-siRNA nanoplex delivery to maintain the integrity of the blood brain barrier. Brain Res. 1282, 142-155 (2009).

9 Simka M. Blood-brain barrier compromise with endothelial inflammation may lead to autoimmune loss of myelin during multiple sclerosis. Curr. Neurovasc. Res. 6(2), 132-139 (2009).

10 Ransohoff RM. Natalizumab for multiple sclerosis. N. Engl. J. Med. 356(25), 2622-2629 (2007).

11 Yan SD, Chen X, Fu J et al. RAGE and amyloid- $\beta$ peptide neurotoxicity in Alzheimer's disease. Nature 382(6593), 685-691 (1996).

12 Deane R, Yan SD, Submamaryan RK et al. RAGE mediates amyloid- $\beta$ peptide transport across the blood-brain barrier and accumulation in brain. Nat. Med. 9(7), 907-913 (2003).

13 Hino $\mathrm{T}$, Yokota $\mathrm{T}$, Ito $\mathrm{S}$ et al. In vivo delivery of small interfering RNA targeting brain capillary endothelial cells. Biochem. Biophys. Res. Commun. 340(1), 263-267 (2006). 
14 Campbell M, Kiang AS, Kenna PF et al. RNAi-mediated reversible opening of the blood-brain barrier. J. Gene. Med. 10(8), 930-947 (2008).

15 Fuest C, Bankstahl M, Winter P, Helm M, Pekcec A, Potschka H. In vivo downregulation of mouse brain capillary P-glycoprotein: a preliminary investigation. Neurosci. Lett. 464(1), 47-51 (2009).

16 Kuwahara H, Nishina K, Yoshida K et al. Efficient in vivo delivery of siRNA into brain capillary endothelial cells along with endogenous lipoprotein. Mol. Ther. 19(12), 2213-2221 (2011).

17 Chen YH, Chang M, Davidson BL. Molecular signatures of disease brain endothelia provide new sites for CNS-directed enzyme therapy. Nat. Med. 15(10), 1215-1218 (2009).

18 Uchida Y, Ohtsuki S, Katsukura Y et al. Quantitative targeted absolute proteomics of human blood-brain barrier transporters and receptors. J. Neurochem. 117(2), 333-345 (2011).

19 Lichota J, Skjørringe T, Thomsen LB, Moos T. Macromolecular drug transport into the brain using targeted therapy. J. Neurochem. 113(1), 1-13 (2010).

20 Kumar P, Wu H, McBride JL et al. Transvascular delivery of small interfering RNA to the central nervous system. Nature 448(7149), 39-43 (2007).
21 Spencer BJ, Verma IM. Targeted delivery of proteins across the blood-brain barrier. Proc. Natl Acad. Sci. USA. 104(18), 7594-7599 (2007).

22 Demeule M, Currie JC, Bertrand Y et al. Involvement of the low-density lipoprotein receptor-related protein in the transcytosis of the brain delivery vector angiopep-2. J. Neurochem. 106(4), 1534-1544 (2008).

23 Gaillard PJ, de Boer AG. 2B-trans technology: targeted drug delivery across the blood-brain barrier. Methods Mol. Biol. 437, 161-175 (2008). 